EPJ Web of Conferences 35, 05007 (2012)

DOI: $10.1051 /$ epjconf/20123505007

(C) Owned by the authors, published by EDP Sciences, 2012

\title{
Reconstructing breakup at sub-barrier energies
}

\author{
D. H. Luong ${ }^{1, \mathbf{a}}$, D. J. Hinde ${ }^{1}$, M. Dasgupta ${ }^{1}$, M. Evers ${ }^{1}$, R. Rafiei ${ }^{1}$, and R. du Rietz ${ }^{1}$
}

Department of Nuclear Physics, Research School of Physics and Engineering, Australian National University, Canberra, Australian Capital Territory 0200, Australia

\begin{abstract}
Using a position sensitive detector array, back-angle coincidence measurements of breakup fragments at sub-barrier energies has enabled the complete characterisation of the breakup processes in the reactions of ${ }^{6,7} \mathrm{Li}$ with ${ }^{208} \mathrm{~Pb}$. Breakup processes and their time-scales are identified through the reaction Q-values and the relative energy of the captured breakup fragments. The majority of breakup processes fast enough $\left(\sim 10^{-22} s\right)$ to affect fusion are triggered by transfer of a neutron from ${ }^{6} \mathrm{Li}$, and of a proton to ${ }^{7} \mathrm{Li}$. These breakup mechanisms should therefore have a major contribution to the $\sim 30 \%$ suppression of complete fusion observed at above-barrier energies.
\end{abstract}

\section{Introduction}

Reactions involving the light nuclei ${ }^{6,7} \mathrm{Li}$ have been studied since the beginning of accelerator-based research into nuclear physics [1]. Nucleon clustering, often in the form of $\alpha$-particles, offers a key insight into explaining the structure of light nuclei. Experiments show that reaction dynamics as well as structure can be strongly affected by clustering, with direct breakup into their constituent clusters expected during nuclear collisions [3-12]. Both ${ }^{6} \mathrm{Li}$ and ${ }^{7} \mathrm{Li}$ have $\alpha+$ residue cluster structures [13], and low inter-cluster binding energies [14] of $1.47 \mathrm{MeV}$ and $2.47 \mathrm{MeV}$ respectively.

Fusion between nuclei is a complex process due to the strong coupling between the relative motion and internal nuclear excitations. Reaction complexity is increased when reacting nuclei have cluster structure with low binding energy, i.e. weakly-bound, since there can be a large breakup probability. Inhibition of above-barrier complete fusion of $34 \%$ was observed for the reaction ${ }^{6} \mathrm{Li}+{ }^{209} \mathrm{Bi}$, and $26 \%$ for ${ }^{7} \mathrm{Li}+{ }^{209} \mathrm{Bi}$, all attributed to cluster breakup of these nuclei [15]. The question still remains "What is the breakup mechanism, and how does this affect near and above barrier fusion for weakly-bound nuclei?"

\section{Experiment}

To experimentally identify and characterise all breakup mechanisms, it is necessary to simultaneously measure both breakup fragments over a wide angular range. We have developed a novel approach, making coincidence measurements of breakup fragments at beam energies below that of the fusion barrier. This suppresses breakup fragment absorption by the target, and allows us to investigate those trajectories that can lead to fusion at higher energies. (a)
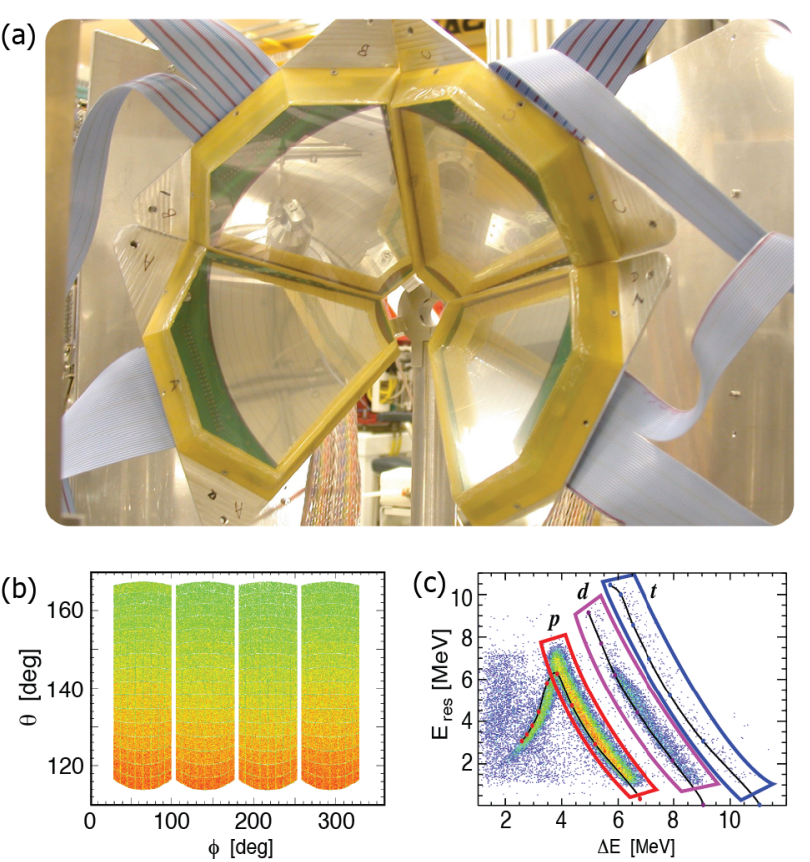

Fig. 1. (a) Photograph of the breakup detector array showing the four DSSDs mounted on a hub in a "lampshade" annular arrangement all angled at $45^{\circ}$, with respect to their bisector, towards the focal point of the hub. (b) The array covers $\sim 50^{\circ}$ in scattering angle $\theta$ and $\sim 300^{\circ}$ in azimuthal angle $\phi$, when arranged in the non-telescopic arrangement. Pixel separation in each detector is exaggerated for clarity. (c) Typical energy loss $A \mathrm{E}$ vs residual energy $\mathrm{E}_{r e s}$ recorded by the detector telescope, for protons (red), deuterons (magenta), and tritons (blue). High- $Z$ particles require much higher energy to pass through the $\triangle \mathrm{E}$ detector and register in $E_{\text {res }}$, e.g. an $\alpha$-particle needs an energy of at least $29.0 \mathrm{MeV}$ to appear in this $\Delta E-E_{r e s}$ spectrum.

\footnotetext{
a e-mail: huy. luong@anu. edu. au
} 
A pixelated detector array was developed at the ANU for the sole purpose of breakup studies. This array consists of four $400 \mu \mathrm{m}$ double-sided silicon strip detectors (DSSDs) with a combined total of 512 position pixels (see figure 1(a)). For measurement of the breakup fragments at backward angles, the array covers $\sim 50^{\circ}$ in scattering angle $\theta$, and $\sim 300^{\circ}$ in azimuthal angle $\phi$, as shown in figure 1(b).

Beams of ${ }^{6,7} \mathrm{Li}$ at $29.0 \mathrm{MeV}$ in the laboratory frame, below the 30.3 and $30.1 \mathrm{MeV}$ barriers for ${ }^{6} \mathrm{Li}+{ }^{208} \mathrm{~Pb}$ and ${ }^{7} \mathrm{Li}+{ }^{208} \mathrm{~Pb}$, respectively, were provided by the Australian National University's 14UD tandem electrostatic accelerator. These bombarded a $98.7 \%$ enriched ${ }^{208} \mathrm{PbS}$ target, $170 \mu \mathrm{g} \mathrm{cm}^{-2}$ in thickness, supported by a $15 \mu \mathrm{g} \mathrm{cm}-2$ carbon backing facing downstream with respect to the beam. Breakup fragments were detected in coincidence at backward angles to avoid the elastically scattered lithium, which is extremely dominant at forward angles. Isotopic identification of high energy singly charge fragments, important for determining the reaction channel, is achieved by re-arranging two DSSD elements back-to-back resulting in a $\Delta E-E_{\text {res }}$ detector telescope. Protons with energy above $7.5 \mathrm{MeV}$, and deuterons and tritons with energy greater than 10.0 and $11.5 \mathrm{MeV}$, respectively, can be cleanly separated as shown in figure 1(c). The identity of other (low energy) coincident charged particles is deduced through kinematic reconstructions of the breakup event. Alpha-particles with energy less than 29.0 MeV cannot pass through the $\Delta E$ detector to register an $E_{\text {res }}$ signal and thus cannot be identified by the $\Delta E-E_{r e s}$ detector telescope.

\section{Binary breakup of ${ }^{6,7} \mathbf{L i}$}

Each breakup event is identified by the kinetic energies, scattering angles and azimuthal angles $\left(E_{i}, \theta_{i}, \phi_{i}\right)$ of the coincident fragments. As an example for the particle identification process, the energies $E_{1,2}$ of the coincident fragment, from the reaction of ${ }^{7} \mathrm{Li}$ on ${ }^{208} \mathrm{~Pb}$ at $29.0 \mathrm{MeV}$, are plotted against each other in figure 2. These events do not involve the back element of the detector telescope, and thus particle species are not known. Immediately obvious are two classes of coincidence event; events with correlated energies $E_{1,2}$ forming diagonal band-like structures, and random coincidence between elastics, noise, and low energy protons, with uncorrelated energies.

The distinct diagonal bands shows coincident particles are being produced from breakup of different projectilelike nuclei. Overlaid, in colour, are events that have one of the two coincident particles isotopically identified by the detector telescope. These include events with an identified deuteron (magenta data points) and events with an identified triton (blue data points), with the other coincident particle being an $\alpha$-particle. Overlapping of identified coincident pairs and the unidentified groups allow identification of the later as breakup events involving $\alpha+d$ and $\alpha+t$ as indicated.

For the remaining correlated coincident pairs, full energy deposition at such high energies implies both particles must be $\alpha$-particles. Having identified both coincident

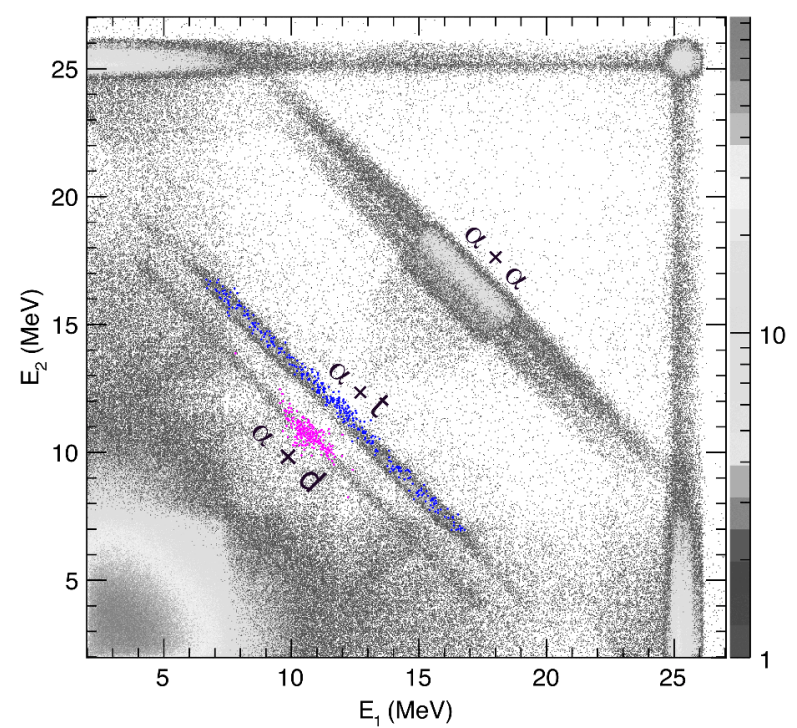

Fig. 2. (color online) Energy correlation between binary coincident particles produced from reaction of ${ }^{7} \mathrm{Li}$ on ${ }^{208} \mathrm{~Pb}$ at $E_{\text {beam }}=29.0 \mathrm{MeV}$. Events comprising the diagonal bands have correlated energies indicating coincident fragments from a common origin. Overlaid are data involving the telescope, allowing identification of breakup of ${ }^{7} \mathrm{Li}$ into $\alpha+t$ (blue) and $\alpha+d$ (magenta). Overlapping between the identified coincident pairs and the unidentified groups allow identification of the later. Coincident pairs forming the last correlated group are identified as $\alpha+\alpha$ based on their energies.

particles, and their energies $E_{1,2}$, further insights into their production process can be obtained through reconstruction of the three-body reaction Q-value.

\subsection{Breakup mechanisms}

From the measured kinetic energies $E_{1,2}$ of the two breakup fragments and the laboratory kinetic energy of the incident projectile $E_{l a b}$ (derived from $E_{\text {beam }}$ after correcting for energy lost in traversing the target), the Q-value was obtained via

$$
Q=E_{1}+E_{2}+E_{r e c}+E_{e x}-E_{l a b} .
$$

Here $E_{e x}$ and $E_{r e c}$ are, respectively, the excitation and recoil energies of the recoiling target-like nucleus, with its mass determined by the masses of the two detected fragments. While both are not measured, $E_{r e c}$ can be determined from conservation of momentum [16].

The reconstructed $Q$-spectra for reactions with ${ }^{6,7} \mathrm{Li}$ at $E_{\text {beam }}=29.0 \mathrm{MeV}$ are shown in figure 3 . At all the measured energies, almost all the yield contributes to sharp peaks in $Q$, meaning the breakup is indeed almost exclusively binary, with identified breakup modes of $\alpha+\alpha$, $\alpha+t, \alpha+d$, and $\alpha+p$. The expected $Q$-values for each binary breakup process, calculated from the masses of all the products, are consistent with the measurements, and indicated for each breakup mode by vertical bars from the $Q$-value axis. 


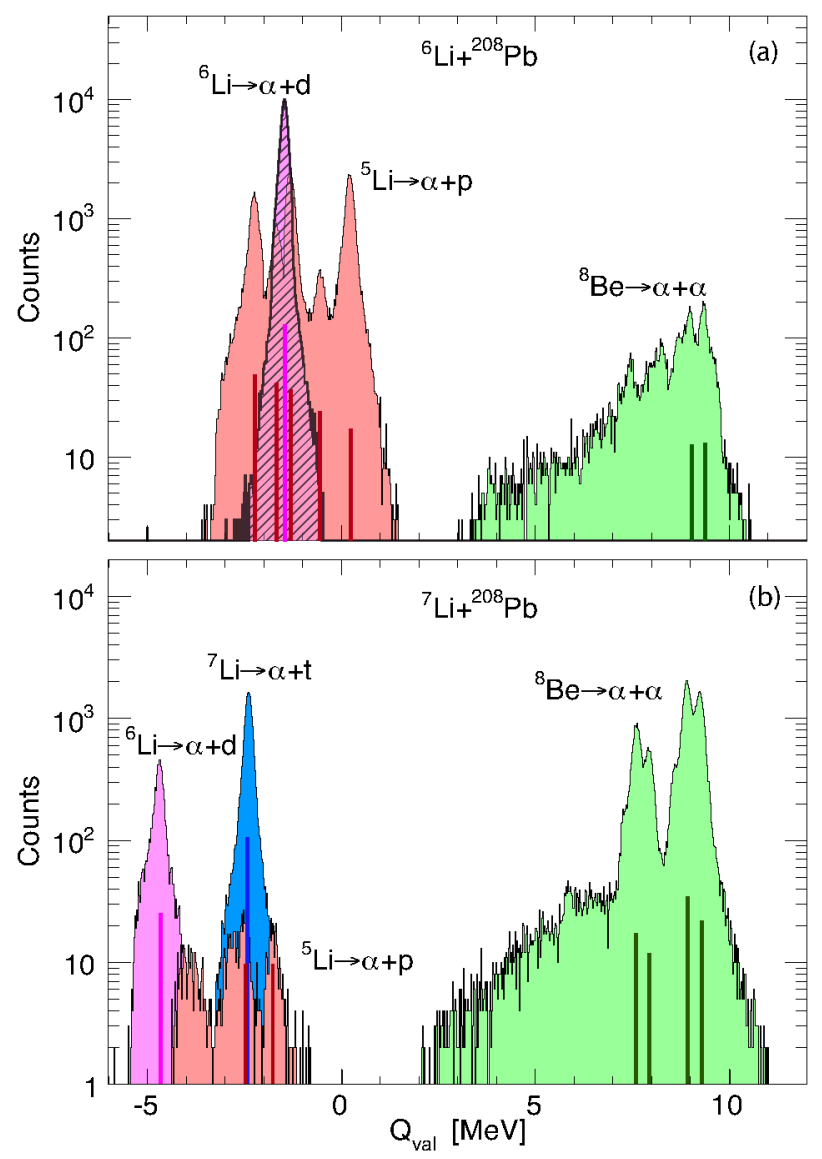

Fig. 3. Measured Q-spectra for the indicated reactions at $\mathrm{E}_{\text {beam }}=29.0 \mathrm{MeV}$. Identified breakup modes, consistent with the calculated Q-values (vertical bars), are indicated for $\alpha+p$ (red), $\alpha+d$ (magenta), $\alpha+t$ (blue), and $\alpha+\alpha$ (green) pairs. The patterned area indicates noise consisting of random coincidences and/or events with incomplete energy deposition. (a) The vertical red bars indicate the Q-values for breakup following the population of five identifiable energy states in ${ }^{209} \mathrm{~Pb}$, the green bars the four lowest energy states in ${ }^{206} \mathrm{Tl}$, and the magenta bar the ${ }^{208} \mathrm{~Pb}$ ground-state. (b) The magenta bars indicate the ${ }^{209} \mathrm{~Pb}$ groundstate, the blue bar corresponds to the ground state of ${ }^{208} \mathrm{~Pb}$, and the green bars indicate the four lowest energy states of ${ }^{207} \mathrm{Tl}$.

For the ${ }^{6} \mathrm{Li}+{ }^{208} \mathrm{~Pb}$ reaction (figure $3(\mathrm{a})$ ), the most intense peak corresponds to ${ }^{6} \mathrm{Li} \rightarrow \alpha+d$ breakup from excited states of the projectile, as might be expected. However, the ${ }^{5} \mathrm{Li} \rightarrow \alpha+p$ breakup contributes to five distinct peaks in the spectrum, matching the expected $Q$-values for neutron stripping from the projectile, and forming the unbound ${ }^{5} \mathrm{Li}$ nucleus and the five identifiable energy states that can be populated in ${ }^{209} \mathrm{~Pb}$. There is also a surprisingly large $\alpha+\alpha$ yield, from breakup following pick-up of a neutron and a proton by the projectile $\left({ }^{6} \mathrm{Li} \rightarrow{ }^{8} \mathrm{Be} \rightarrow \alpha+\alpha\right)$.

For the ${ }^{7} \mathrm{Li}+{ }^{208} \mathrm{~Pb}$ reaction (figure $3(\mathrm{~b})$ ), direct breakup into $\alpha+t$ is prominent as expected. However, yield in ${ }^{8} \mathrm{Be} \rightarrow \alpha+\alpha$, following the ${ }^{208} \mathrm{~Pb}\left({ }^{7} \mathrm{Li},{ }^{8} \mathrm{Be}\right){ }^{207} \mathrm{Tl}$ proton pick-up reaction, is much more likely. The $Q$-spectrum shows that ${ }^{207} \mathrm{Tl}$ is populated mainly in its four lowest energy states. There is a small yield corresponding to
${ }^{6} \mathrm{Li} \rightarrow \alpha+d$ breakup, triggered by stripping of a neutron from the projectile forming ${ }^{6} \mathrm{Li}$. An insignificant yield attributed to ${ }^{5} \mathrm{Li} \rightarrow \alpha+p$ breakup, following two neutron stripping of the projectile to make the ${ }^{5} \mathrm{Li}$ nucleus, can also be seen.

Identification of the reaction processes leading to breakup is not sufficient to understand the interplay between breakup and suppression of fusion [5]. It is critical to also know whether the breakup occurs before or after the projectile reaches its point of closest approach to the target nucleus. Consider the ${ }^{6} \mathrm{Li} \rightarrow \alpha+d$ breakup from resonance states in ${ }^{6} \mathrm{Li}$. The long lifetime [17] of $2.7 \times$ $10^{-20} \mathrm{~s}$ in the $3^{+}(2.18 \mathrm{MeV})$ resonance, as compared to the $10^{-22} \mathrm{~s}$ time-scale for the direct reaction, means ${ }^{6} \mathrm{Li}$ in this resonance state would survive intact hence can participate in reactions at energies above the fusion-barrier. Therefore the ${ }^{6} \mathrm{Li} \rightarrow \alpha+d$ breakup from such resonant states will not compete with complete fusion.

To separate breakup that may compete directly with complete fusion (prompt breakup) from slower breakup processes (such as breakup from long-lived resonances) requires information on the excitation of the parent nuclei. This important information can be recovered from the relative energy between the breakup fragments, and is directly related to the time-scale of breakup [18] .

\subsection{Time-scale of breakup}

If we consider nuclear collisions at energies below the fusion-barrier energy from a classical perspective, we can picture the Coulomb field associated with the target nucleus as a spherical mirror. A projectile approaching the target nucleus can be excited to excitation energy $E^{*}$, and may breakup after reflection, far away from the point of closest approach. Such breakup is termed asymptotic breakup and, with minimal interaction with the target nucleus following breakup, the relative energy $E_{\text {rel }}$ between the breakup fragments will comprise

$$
E_{r e l}=E^{*}+Q_{B U}
$$

where $Q_{B U}$ is the breakup $Q$-value. Breakup of the projectile can also happen before reflection, as it advances towards the target, with each charged breakup fragment experiencing different acceleration by the Coulomb field of the target nucleus [19-21]. These events are termed prompt breakup, with the relative energy $E_{\text {rel }}$ now comprising an extra term

$$
E_{\text {rel }}=E^{*}+Q_{B U}+E_{\text {post_acc. }} .
$$

This quantitative dependence of $E_{r e l}$ on breakup trajectories, or internuclear separation at breakup, has been shown $[17,18]$, and is related to the breakup time-scale [16, 17]. Furthermore, discrete peaks in the $E_{\text {rel }}$ distribution have been shown $[17,18]$ to come from asymptotic breakup from the decay of long-lived states of the projectile, while events broad distribution in $E_{r e l}$ comprise prompt breakup from all trajectories. 


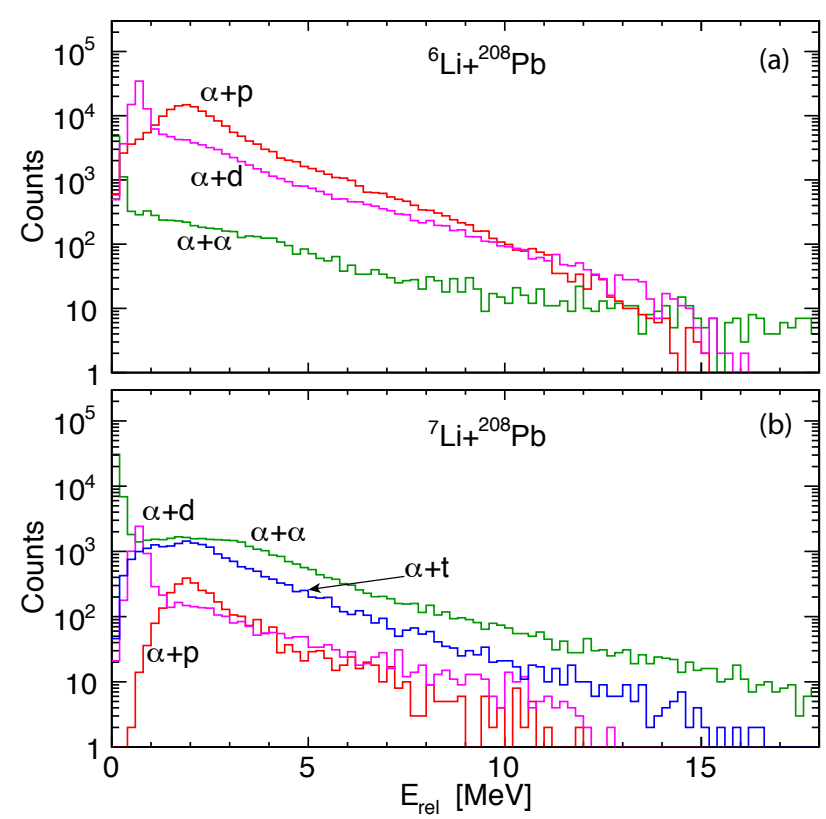

Fig. 4. Experimental $E_{r e l}$-spectra for breakup of ${ }^{6,7} \mathrm{Li}$ into $\alpha+p$ (red), $\alpha+d$ (magenta), $\alpha+t$ (blue), and $\alpha+\alpha$ (green) while in collisions with ${ }^{208} \mathrm{~Pb}$ at $E_{\text {beam }}=29.0 \mathrm{MeV}$. The peak at $0.7 \mathrm{MeV}$ for $\alpha+d$ breakup corresponds to decay from the $3^{+}(2.18 \mathrm{MeV})$ resonant state in ${ }^{6} \mathrm{Li}$. With a lifetime of $2.7 \times 10^{-20} \mathrm{~s}$, breakup from this state is too slow to compete with direct reactions. The low energy peak at $92 \mathrm{keV}$, for ${ }^{8} \mathrm{Be} \rightarrow \alpha+\alpha$, results from the ground state decay of ${ }^{8} \mathrm{Be}$. The yield at high $E_{r e l}$ for the $\alpha+\alpha$ breakup shows that breakup from excited ${ }^{8} \mathrm{Be}$ is dominant in the ${ }^{7} \mathrm{Li}$-induced reaction.

\subsection{Separation of prompt and delayed breakup}

Experimentally $E_{r e l}$ can be determined from energies $E_{1,2}$, the deduced masses $m_{1,2}$, and the angular separation $\theta_{12}$ of the coincident fragments

$$
E_{r e l}=\frac{m_{2} E_{1}+m_{1} E_{2}-2 \sqrt{m_{1} E_{1} m_{2} E_{2}} \cos \theta_{12}}{m_{1}+m_{2}}
$$

Shown in figure 4 are the experimental $E_{r e l}$, as determined using equation (4), for the reactions with ${ }^{6,7} \mathrm{Li}$ at beam energy of $29.0 \mathrm{MeV}$. These spectra have not been corrected for the detector efficiency, however, loss due to punch through of high energy singly charged particles hitting the non-telescope section of the detector has been accounted for.

Examination of the peaks at low $E_{\text {rel }}$ from both reactions reveals that ${ }^{8} \mathrm{Be} \rightarrow \alpha+\alpha$ breakup shows a sharp peak at $E_{\text {rel }} \approx 92 \mathrm{keV}$ corresponding to the slow, $\sim 10^{-16} \mathrm{~s}$, ground-state decay of ${ }^{8} \mathrm{Be}$. This comprises $\sim$ half of all the $\alpha+\alpha$ yield. For breakup into $\alpha+d$, the peak at $0.7 \mathrm{MeV}$ corresponds to the decay of the first resonant state in ${ }^{6} \mathrm{Li}$, with a relatively long lifetime of $2.7 \times 10^{-20} \mathrm{~s}$. It is populated by direct excitation of ${ }^{6} \mathrm{Li}$ (figure $4(\mathrm{a})$ ) or through n-transfer in the ${ }^{7} \mathrm{Li}$ reaction (figure 4(b)). The long life-time of the resonant state implies that $\alpha+d$ breakup events associated with these peaks happens far from the target nucleus, in the asymptotic region. Hence these $\alpha+d$ breakup events will not compete with complete fusion at beam energies above that of the barrier-energy.

At higher $E_{r e l}$, for the ${ }^{6} \mathrm{Li}$ reaction, the ${ }^{5} \mathrm{Li} \rightarrow \alpha+p$ breakup is very significant. It arises from breakup following neutron transfer and makes the largest contribution to prompt breakup in the reaction with ${ }^{6} \mathrm{Li}$. The remainder is prompt $\alpha+d$ breakup. For the reaction with ${ }^{7} \mathrm{Li}$, the ${ }^{7} \mathrm{Li} \rightarrow \alpha+t$ breakup is prominent, with a wide $E_{r e l}$ distribution consistent with prompt breakup. The largest contribution to prompt breakup of ${ }^{7} \mathrm{Li}$, however, is from the prompt component of ${ }^{8} \mathrm{Be} \rightarrow \alpha+\alpha$ (those $\alpha+\alpha$ coincidences with high $\left.E_{r e l}\right)$.

\section{Conclusion}

Back-angle coincidence measurements of the reactions induced by ${ }^{6,7} \mathrm{Li}$ on ${ }^{208} \mathrm{~Pb}$, at sub-barrier energies, have revealed the mechanisms triggering breakup of these weakly-bound nuclei. Using the well-accepted $\alpha+$ deuteron and $\alpha+$ triton cluster structure, one might expect direct breakup into the cluster partners would be the dominant breakup channel for ${ }^{6} \mathrm{Li}$ and ${ }^{7} \mathrm{Li}$, respectively. This, in fact, turns out to be one of the weaker breakup channels. Breakup of ${ }^{6,7} \mathrm{Li}$, as seen here, is frequently a multistep process initiated by transfer and is thus target dependent. Even multi-nucleon transfer to and from ${ }^{6,7} \mathrm{Li}$, triggering breakup has been observed. These measurements in conjunction with classical model calculations are expected to lead to a more quantitative understanding of the breakup mechanism and its effects on fusion.

\section{References}

1. J. D. Cockcroft, Nobel Lecture (1951).

2. W. von Oertzen et al., Phys. Rep. 432, 43 (2006).

3. M. Dasgupta et al., Phys. Rev. Lett. 82, 1395 (1999).

4. D. J. Hinde et al., Phys. Rev. Lett. 89, 272701 (2002).

5. M. Dasgupta et al., Phys. Rev. C 70, 024606 (2004).

6. E. Speth et al., Phys. Rev. Lett. 24, 1493 (1970).

7. D. L. Disdier et al., Phys. Rev. Lett. 27, 1391 (1971).

8. O. Häusser et al., Phys. Lett. B 38, 75 (1972).

9. R. Ost et al., Phys. Rev. C 5, 5 (1972).

10. K. O. Pfeiffer et al., Nuc. Phys. A 206, 545 (1973).

11. H. Freiesleben et al., Phys. Rev. C 10, 245 (1974).

12. R. Ost et al., Z. Phys. 266, 369 (1974).

13. G. C. Morrison, Phys. Rev. Lett. 5, 565 (1960).

14. D. R. Tilley et al., Nuc. Phys. A 708, 3 (2002).

15. M. Dasgupta et al., Phys. Rev. C 66, 041602(R) (2002).

16. R. Rafiei et al., Phys. Rev. C 81, 024601 (2010).

17. D. H. Luong et al., Phys. Lett. B 695, 105 (2011).

18. D. H. Luong et al., EPJ Web of Conf. 17, 03002 (2011).

19. G. Baur et al., Nuc. Phys. A 504, 352 (1989).

20. J. E. Mason et al., Phys. Rev. C 45, 2870 (1992).

21. C. A. Bertulani, Phys. Rev. C 49, 2688 (1994). 